\title{
Aplicación del modelo 4MAT y su influencia en el rendimiento académico de cinemática en estudiantes universitarios
}

\section{Application of the 4MAT model and its influence in the performance of kinematics course in university students}

\author{
Edilberto Valencia Salazar ${ }^{1}$, Segundo E. Vergara Medrano², Luis O. Carbajal García y Manuel J. Sánchez \\ Chero $^{3}$
}

\section{RESUMEN}

Se presentan resultados de la aplicación del Modelo 4MAT en el marco del estudio sobre estilos de aprendizaje y desempeño en la enseñanza de Cinemática del curso de Cinemática de Ingeniería Agroindustrial de la Universidad Nacional Intercultural de la Amazonía. Para lograrlo, se elaboró estrategias de enseñanza aprendizaje basados en los ocho pasos del Modelo 4MAT. Esta experiencia estuvo basada en el enfoque cuantitativo, de tipo aplicativa con diseño cuasi experimental. La muestra consistió de 36 estudiantes (18 del Grupo Experimental y 18 del Grupo Control) de una población de 250. El análisis de resultados se realizó con pruebas, T de Student y Wilcoxon. Se demostró que la aplicación del Modelo 4MAT influye significativamente en el rendimiento académico de estudiantes del ciclo II de la Universidad, aseveración que se sustenta en el análisis estadístico de T de Student, los estudiantes del grupo experimental obtuvieron mejores notas (Promedio 10,44) después del experimento respecto a los del grupo control (Promedio $=5,89$ ); por lado, la significancia estadística $\mathrm{p}=0,00<0,05=\alpha$ además $\left(\mathrm{t}_{\mathrm{c}}=1\right.$, $\left.154<\mathrm{t}_{\mathrm{t}}=1,74\right)$, significó aceptar la hipótesis de investigación. La aplicación del Modelo 4MAT influye también significativamente en cuanto al cambio actitudinal positivo en los estudiantes.

Palabras clave: enseñanza-aprendizaje, Modelo 4MAT, Cinemática, Rendimiento Académico.

\begin{abstract}
The results of the application of the 4MAT Model are presented in the framework of the study on learning styles and performance in the teaching of Kinematics of the course of Agroindustry Engineering of the Universidad Nacional Intercultural de la Amazonía. To achieve this, teaching-learning strategies were elaborated based on the eight steps of the 4MAT Model. This experience was based on the quantitative approach, of application type with the quasi-experimental design. The sample consisted of 36 students (18 from the Experimental Group and 18 from the Control Group) from a population of 250. The analysis of results was carried out with tests, Student's T and Wilcoxon. It was demonstrated that the application of the Model 4MAT, does influence the academic performance of students of Cycle II of the University significantly, assertion that is based on the statistical analysis of Student's T. The students of the experimental group which obtained better scores (Average 10.44) after the experiment with respect to those of the control group (Average $=5.89$ ); on the other hand, the statistical significance $p=0,00<0.05$ $=\alpha$ plus $\left(t_{c}=1,154<t_{t}=1,74\right)$, meant accepting the research hypothesis. The application of the 4MAT Model also significantly influences the positive attitudinal change in the students.
\end{abstract}

Keywords: teaching-learning, 4MAT Model, Kinematics, Academic Performance.

\footnotetext{
Universidad Nacional Intercultural de la Amazonia. edivasa@hotmail.com

2Universidad Nacional de Jaén. svergara@unj.edu.pe, carbajal@hotmail.com

${ }^{3}$ Universidad Nacional de Frontera
} 


\section{INTRODUCCIÓN}

La Física es una ciencia fundamental. Como curso se ubica entre las materias con mayor dificultad para aprobarla por los estudiantes. Esto se debe a muchos factores: la dedicación que se necesita para su comprensión y dominio, la estigmatización como curso difícil y el rol de docentes con poco interés por innovar con nuevas metodologías de enseñanza aprendizaje y que siguen enseñando como ellos fueron instruidos (metodología tradicional), es decir, no se preocupan por mejorar la interacción con el estudiante y la forma particular como ellos aprenden. Esta dificultad es uno de los factores principales en los altos índices de desaprobación y deserción de estudiantes en los primeros ciclos de las Carreras de Ciencia e Ingeniería en muchas universidades de Latinoamérica y, en particular, de Ucayali. Estos aspectos son razones suficientes para buscar nuevas formas de enseñar la Física a nivel universitario. Pues se sabe que los cursos de Física son materias básicas en las carreras de Ciencia e Ingeniería y son, además, los pilares sobre los cuales se apoyan los demás asignaturas en ciclos más avanzados.

Por otro lado, muchos investigadores, en el campo educativo, han demostrado que la enseñanza bajo nuevos métodos ha mejorado el rendimiento académico y la predisposición de estudiantes al aprendizaje de alguna materia, por ejemplo, la aplicación del Software (SAGE) en Mecánica de Fluidos (Pantoja, 2015); la aplicación del Sistema Multimedia Interactivo (SAMI) (Marzano, 2014) y la aplicación del Sistema 4MAT en Física (Ramírez, 2009; Rosado, y Sánchez, 2012; Artomónova et al., 2014); y otras disciplinas (Nicoll y Seider, 2010; Dikkartin, 2012; Nowacki, 2011; Tatar y Dikici, 2009). En forma análoga se ha estudiado un tema relacionado con este trabajo, por ejemplo, la relación entre los estilos de aprendizaje del estudiante y el rendimiento académico (Leonardo y Tarazona, 2014; Ramírez et al., 2013). El bajo rendimiento académico y la deserción en la educación universitaria puede deberse a muchos factores: académico, social, económico, etc. (Mori, 2012). En consecuencia, lo que se pretende es mejorar el desempeño académico del estudiante a partir de la mejora del factor académico. Por razones de alcance de este trabajo de investigación, y considerando que dentro del factor académico tenemos otros subfactores, por ejemplo: ambientes inadecuados para el estudio, los estudiantes no se adecúan rápidamente a la nueva vida universitaria, manejo inadecuado de los recursos TICs, bibliotecas implementadas inadecuadamente, metodologías incorrectas en los planes curriculares, no existen proyectos educativos, los docentes no implementan metodologías actualizadas en la enseñanza- aprendizaje de acuerdo a los avances en la educación, entre otras.

Por tanto existen razones suficientes para plantear la siguiente investigación ¿Cómo la aplicación del Modelo 4MAT influye en el rendimiento académico del curso de Cinemática en estudiantes universitarios? y específicamente ¿Cómo la aplicación del Modelo 4MAT influye en el rendimiento conceptual, procedimental y actitudinal?; lo cual conlleva a plantearnos la siguiente hipótesis: "La aplicación del Modelo 4MAT influye en el rendimiento académico de Cinemática en estudiantes universitarios" y específicamente "La aplicación del Modelo 4MAT influye en el rendimiento: conceptual, procedimental y actitudinal de estudiantes universitarios. Para demostrar estas hipótesis se planteó los objetivos: demostrar que la aplicación del Modelo 4MAT influye en el rendimiento académico de Cinemática en los estudiantes universitarios, y en los casos específicos, demostrar que la aplicación del Modelo 4MAT influye en el rendimiento conceptual, procedimental y actitudinal de Cinemática en los estudiantes universitarios.

\section{EI Modelo 4MAT.}

El Modelo 4MAT se fundamenta en: la teoría de la Pedagogía Instrumentalista de John Dewey, la teoría del Aprendizaje Experimental de David Kolb y la teoría sobre los Tipos de Personalidad de Carl Jung. El Modelo 4MAT fue desarrollado por Bernice McCarthy en 1987. Es una modificación de la Teoría de Kolb. Bernice McCarthy así, organiza en su modelo, la enseñanza-aprendizaje de manera que exista un equilibrio de las tensiones entre las situaciones concretas experimentales y conceptuales abstractas. La calidad y profundidad comprensivas de estos conjuntos de aprendizaje, especialmente sus articulaciones en lenguaje práctico, son las contribuciones de McCarthy a la expansión de la teoría de Kolb. Podríamos decir que en el Modelo 4MAT se distinguen claramente tres elementos:1) los estilos de aprendizajes, 2) el enfoque cerebral y 3) las modalidades estructuradas en un ciclo de aprendizaje. En cuanto a los estilos de aprendizaje, se distinguen cuatro tipos de estudiantes: imaginativos, analíticos, sentido común y dinámicos; en el segundo elemento sobre el enfoque cerebral, es uno de los aportes de McCarthy que es utilizados en la construcción del Modelo 4MAT, basado en investigaciones de la época (1985) sobre el funcionamiento cerebral. Por aquella época se tenía los aportes realizados por Spirry (1961) que dio origen a la Teoría del Cerebro Derecho versus el Cerebro Izquierdo (McCarthy y McCarthy, 2006). 
Actualmente existen otros enfoques cerebrales, por mencionar algunos: la Teoría del Cerebro Triuno de Macleam (1978), Modelo del Cerebro Total de Herrmann (1989), etc. (Segarra et al., 2015). No es el objetivo de la presente investigación, pero queda como propuesta para trabajos futuros sea de Maestría o Doctorado aplicar estos nuevos enfoques que existen o que se descubrirán en el futuro y mejorar el Modelo 4MAT (Muñoz et al., 2015)

Podemos emplear deliberadamente técnicas de enseñanza para excitar el sistema cerebro-mente. Al marco teórico que utilizamos para esta excitación intencional del cerebro-mente le llamamos modalidades de aprendizaje. Aunque hay una gran variedad de modalidades como excitar el sistema cerebro-mente, hemos reducido la lista a los siguientes: Simbólico / abstracto, visual, auditiva y kinestésicos. Cada uno de estas maneras se basa en la investigación de las neurociencias. La investigación apoya que el cerebro se excita en diferentes patrones en respuesta a diferentes entradas fisiológicas (Ramírez, 2009; Ramírez, 2010; Ramírez y Chávez, 2012).

McCarthy opina que habitualmente los colegios dan énfasis sólo a las áreas de adquisición del conocimiento y aplicaciones generales, estilo Analítico y estilo Sentido Común, respectivamente. Para poder subsanar esta necesidad de enseñanzaaprendizaje, McCarthy crea una serie de pasos que ayudan a los docentes a preparar sus clases. Aquí describimos la guía de ocho (8) pasos para la construcción general del ciclo de aprendizaje orientado hacia la ciencia (Rosado, 2011):

\section{Primer cuadrante-paso uno-hemisferio derecho.}

En este paso, hay que proponer una experiencia concreta relacionada al tema, de manera que los estudiantes sean capaces de ver las conexiones con su propia experiencia. El método es la lluvia de ideas en debate.

\section{Primer cuadrante-paso dos-hemisferio izquierdo.}

En este paso, el docente da el primer acercamiento al concepto, deberá involucrar los conocimientos y experiencias expuestas por los estudiantes en el paso anterior. Se recomienda una actividad reflexiva.

\section{Segundo cuadrante-paso tres-hemisferio derecho}

En este paso, se debe hacer la construcción de una imagen que permita visualizar lo reflexionado anteriormente, las imágenes y los símbolos permiten al estudiante llevar su experiencia al desarrollo de un pensamiento reflexivo. Se deberá programar una actividad formal, ya sea esquematizado con audiovisuales o diagramas, mapas mentales o conceptuales.

\section{Segundo cuadrante-paso cuatro- hemisferio izquierdo.}

Aquí es donde el estudiante dispondrá de la información relacionada con el concepto antes de comprenderlo en las maneras convencionales. Es aquí donde se enfatiza en el análisis de los conceptos, hechos, generalizaciones y teorías. Para llevar a cabo este momento, el docente se deberá apoyar en algunos recursos como las lecturas, los textos, resolución de problemas.

\section{Tercer cuadrante-paso cinco-hemisferio izquierdo}

El estudiante evoluciona a lo aprendido en los pasos anteriores. Hacen hojas de trabajo, leen libros de texto, etc. Estos materiales se utilizan para reforzar el concepto y las habilidades enseñadas en el cuadrante 2. El profesor se encargará de revisar esta aplicación, a través de problemas, ejemplos, situaciones de vida, etc. y asegurarse de que lo aprendido es lo correcto, si no es así, deberá realizar una retroalimentación para que el estudiante logre el objetivo.

\section{Tercer cuadrante-paso seis-hemisferio derecho}

En este paso, el estudiante podrá desarrollar sus propias aplicaciones demostrando que es capaz de utilizar lo aprendido en su propio ambiente y contexto. Se recomienda realizar una actividad que permita, a través de la experiencia, reflexionar sobre la práctica.

\section{Cuarto cuadrante-paso siete-hemisferio izquierdo}

En este paso, los estudiantes están obligados a organizar y analizar lo que han aprendido de alguna manera personal. Es aquí donde se perfecciona lo aprendido. El estudiante debe ubicar en su vida cotidiana la utilidad y la prioridad de lo que acaba de aprender. Se recomienda el desarrollo de una actividad que permita experimentar y aplicar los conocimientos en la vida cotidiana y en el contexto habitual (ensayo).

\section{Cuarto cuadrante-paso ocho-hemisferio derecho}

En este paso, los estudiantes deben compartir lo que han aprendido con los demás, mediante trabajos o publicaciones que puedan mostrar a través de diversos medios. Se les alienta a asumir la responsabilidad de hacer su propio sentido de lo que han aprendido para su aplicación en la vida. La culminación de este paso es extender el sentido de haber aprendido el concepto para su aplicación en la vida.

En la Figura1, se muestra el ciclo de enseñanza- 
aprendizaje del Modelo 4MAT descrito previamente.

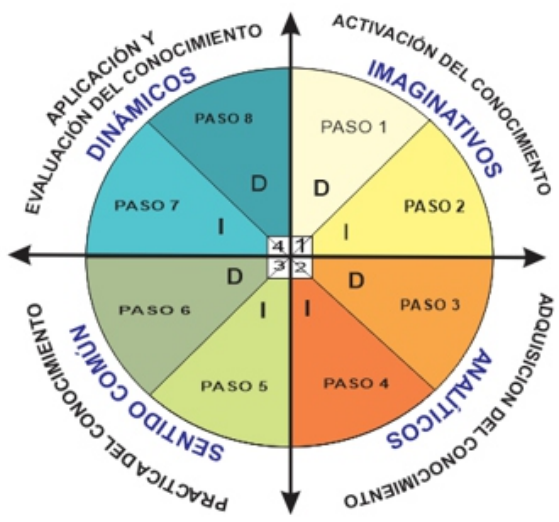

Figura 1. El Modelo 4MAT

\section{Rendimiento Académico.}

Tanto Campana como el Ministerio de Educación, se refieren al rendimiento académico como el nivel de conocimiento expresado en una nota numérica que obtiene el estudiante como resultado de una evaluación, que mide el producto del proceso enseñanza-aprendizaje en el que participa. Es lograr, la máxima eficiencia en el nivel educativo donde el estudiante puede demostrar sus cualidades cognitivas, conceptuales, actitudinales y procedimentales (Pantoja, 2015; Vizconde, 2009).

\section{MATERIAL Y MÉTODOS}

Se ha construido un ciclo de aprendizaje de acuerdo a la guía dada por (McCarthy, 1985), para el tema de Cinemática, la misma que fue aplicada a los estudiantes del curso de Física I de la escuela Académica Profesional de Ingeniería Agroindustrial.

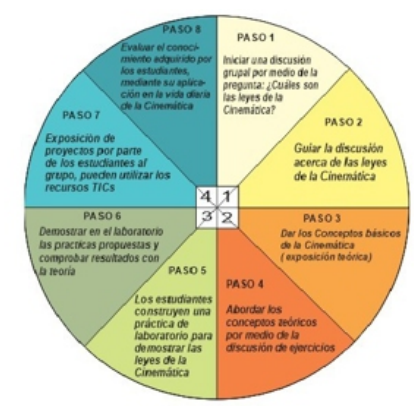

Figura 2. La enseñanza-aprendizaje de la Cinemática en base a los ocho (8) pasos del Modelo $4 M A T$.

En el problema se ha identificado dos variables de investigación:

Variable independiente: Aplicación del Modelo 4MAT.

La variable, Aplicación del Modelo 4MAT, ha sido dimensionada como: Activación del conocimiento, adquisición del conocimiento, práctica del conocimiento y aplicación y evaluación del conocimiento; para su desarrollo se elaboró 8 sesiones (ver Figura 2) de enseñanza aprendizaje y luego se aplicó a los estudiantes del grupo experimental, la aplicación se desarrolló con los estudiantes del primer semestre 2017-I, del Ciclo II de la Escuela Académico Profesional de Ingeniería Agroindustrial en la Facultad de Ingeniería y Ciencias Ambientales de la Universidad Nacional Intercultural de la Amazonía.

\section{Variable dependiente: Rendimiento Académico.}

La variable, Rendimiento Académico, con el objeto de alcanzar los objetivos de este trabajo, operacionalmente ha sido dimensionada como: Conceptual, Procedimental y Actitudinal; para su valoración se basó en la técnica de la prueba objetiva (Pre prueba y Pos Prueba), y un test para la parte actitudinal en la entrada y salida, a los estudiantes del Grupo Experimental y del Grupo de Control.

Para esta investigación se empleó el diseño cuasi experimental con Pre Prueba y Pos Prueba y grupos intactos (uno de ellos de control) no aleatorios debido a que la aleatorización no fue factible por lo mencionado en párrafo anterior, por lo que se procuró emplear grupos que sean los más equivalentes posibles al inicio del estudio, formados antes del experimento, son grupos intactos (Hernández et al., 2014).

La población de estudio estuvo integrada por 36 estudiantes del Ciclo II del curso de Física I, de la Escuela Académico Profesional de Ingeniería Agroindustrial. En este sentido, se trabajó con una muestra no probabilística intencionada conformada de la siguiente manera, ver tabla 1:

Tabla 1. Distribución muestral por grupos de estudio

\begin{tabular}{|c|c|c|c|}
\hline \multirow{2}{*}{$\begin{array}{l}\text { Carrera } \\
\text { Profesional de }\end{array}$} & \multicolumn{2}{|c|}{ Distribución } & \multirow{2}{*}{$\begin{array}{c}\text { Total de } \\
\text { estudiantes }\end{array}$} \\
\hline & Aula & Aula & \\
\hline Ingeniería & A & B & \\
\hline \multicolumn{4}{|l|}{ Agroindustrial } \\
\hline GE: Grupo & 18 & $\begin{array}{l}------ \\
\end{array}$ & 18 \\
\hline \multicolumn{4}{|l|}{ Experimental } \\
\hline \multicolumn{4}{|l|}{ Control } \\
\hline & & & 36 \\
\hline
\end{tabular}

En la presente investigación se tuvo en cuenta como primer paso la revisión de los instrumentos para la recolección de datos. Se elaboraron dos Pruebas objetivas (Pre Prueba y Pos Prueba) de 30 ítems cada una con los temas de Cinemática donde evaluamos lo conceptual y procedimental. Para la parte actitudinal 
ha sido adaptado del test (Pantoja, 2015). Para la validación del instrumento se empleó la técnica denominada juicio de expertos. Para su confiabilidad los cuestionarios fueron sometidos primero a la prueba de fiabilidad de Kuder Richarson para una muestra piloto de 7 estudiantes de otra universidad que ya habían llevado el tema de Cinemática; mostrando que tiene una excelente confiabilidad de 0,799 . Esto nos garantizó que el instrumente es aplicable y puede ser aplicable al estudio. De igual forma se procedió con la prueba actitudinal mostrando una confiabilidad de 0,873 , en forma análoga mostrando que es bastamente confiable.

\section{Tratamiento estadístico}

Después de tomadas las pruebas (Pre Prueba y Pos Prueba) se revisaron y calificaron, se hizo una base de datos y se procedió al procesamiento con el programa SPSS versión 22 para Microsoft en español. Por ser la presente investigación de naturaleza no experimental, la prueba de hipótesis se realizó con la T de Student y Wilcoxon (Mendenhall et al., 2010), debido a los resultados de la prueba de normalidad.

\section{Procedimiento}

En el presente trabajo se siguieron los siguientes pasos a fin de probar las hipótesis formuladas:

Se determinó el nivel de significancia con que se trabajó: $\mathrm{p}($ valor $)=\mathrm{p}<0,05=\alpha$.

Se efectuó la prueba de normalidad con el test estadístico de Shapiro-Wilk (por ser una muestra de 18 elementos menor a 30) para determinar el tipo de prueba estadísticas a utilizar, si es paramétricas o no paramétricas. En la presente investigación se utilizaron la prueba estadística paramétrica $\mathrm{T}$ de Student y la prueba no paramétrica de Wilcoxon. Se determinó si la prueba estadística arroja resultados en la zona de rechazo o en la de no rechazo.

Se contrastaron los resultados con las fuentes teóricas o investigaciones antecedentes y los valores tabulados para la prueba $\mathrm{T}$ de Student para 17 grados de libertad, el cual es 1.74 .

\section{RESULTADOS}

\section{Rendimiento Académico.}

Los resultados estadísticos de la Pre Prueba en ambos grupos de estudio, fueron similares en cuanto al nivel de conocimiento previo en Cinemática, la significancia estadística $\mathrm{p}=0,929>0,05=\alpha$, además $\left(\mathrm{t}_{\mathrm{c}}=-0,090<\mathrm{t}_{\mathrm{t}}=1,74\right)$, es diferente al $95 \%$ de confiabilidad de acuerdo a la prueba paramétrica $T$ de Student. También observamos que si comparamos las medias de los resultados de la Pre Prueba de ambos grupos, hay una ligera ventaja de los estudiantes del Grupo de experimental de 0,06 respecto a los estudiantes del Grupo de Control. Por lo tanto, no es significativa la diferencia entre ambos grupos.

De los resultados estadísticos de la Pos Prueba en ambos grupos de estudio, los estudiantes a quienes se les aplicó el Modelo 4MAT, Grupo Experimental, obtuvieron notas superiores (Promedio $=10,44$ ) frente al Grupo de Control (Promedio=6,94), por lo cual proviene el grado de significancia estadística $\mathrm{p}=$ $0,00<0,05=\alpha$, además $\left(\mathrm{t}_{\mathrm{c}}=5,672>\mathrm{t}_{\mathrm{t}}=1,74\right)$; esto significa rechazar la hipótesis nula y aceptar la hipótesis alterna. También observamos que si comparamos las medias de los resultados de la Pos Prueba de ambos grupos, hay una ventaja de los estudiantes del Grupo de Experimental de 3.5 respecto a los estudiantes del Grupo de Control. Por lo tanto, es significativa la influencia de la aplicación del Modelo 4MAT en el Rendimiento Académico en Cinemática, en los estudiantes. La Figura 3 complementa estos resultados.

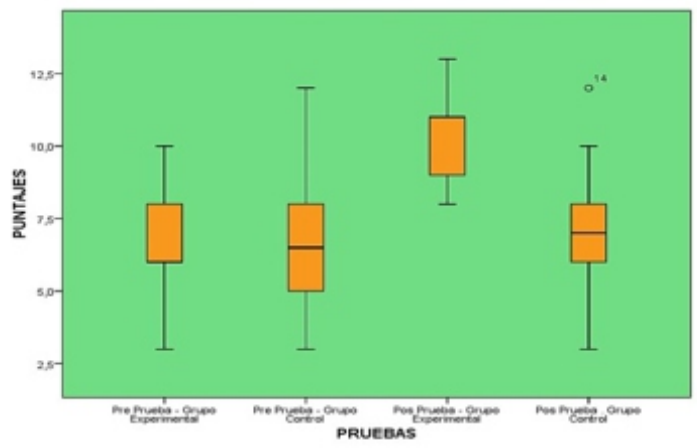

Figura 3. Rendimiento Académico en Cinemática

\section{Rendimiento Conceptual}

Los resultados estadísticos de la Pre Prueba en cada grupo de estudio, se observa que en los estudiantes de ambos grupos antes de iniciarse el experimento presentan resultados ligeramente diferentes en cuanto al nivel de conocimiento Conceptual de Cinemática, por lo que se tiene que el grado de significancia estadística $p=0,154>0,05=$, además $\left(t_{c}=1,492<t_{t=} 1,74\right)$, es distinto al $95 \%$ de confiabilidad de acuerdo a la prueba $\mathrm{T}$ de Student. También se observa una diferencia en la media de 0,56 del Grupo de Control respecto al Grupo Experimental. Por lo tanto, se observa que no es significativa la diferencia entre ambos grupos.

Así mismo, si observamos los estadísticos de la Pos Prueba de ambos grupos de estudio, los estudiantes a quienes se les aplicó el Modelo 4MAT Grupo 
Experimental obtuvieron notas superiores (Promedio $=5,89)$ frente al Grupo de Control (Promedio $=4,33$ ), de lo cual proviene el grado de significativa estadística $p=0,00<0,05=$ además $\left(t_{c}=3,242>t_{t}=1,74\right)$, esto significa rechazar la hipótesis nula y aceptar la hipótesis alterna. También se observa una diferencia en la media de 1,56 del Grupo de Experimental respecto al Grupo de Control. Por tanto, es significativa la diferencia entre ambos grupos. La figura 4 complementa estos resultados.

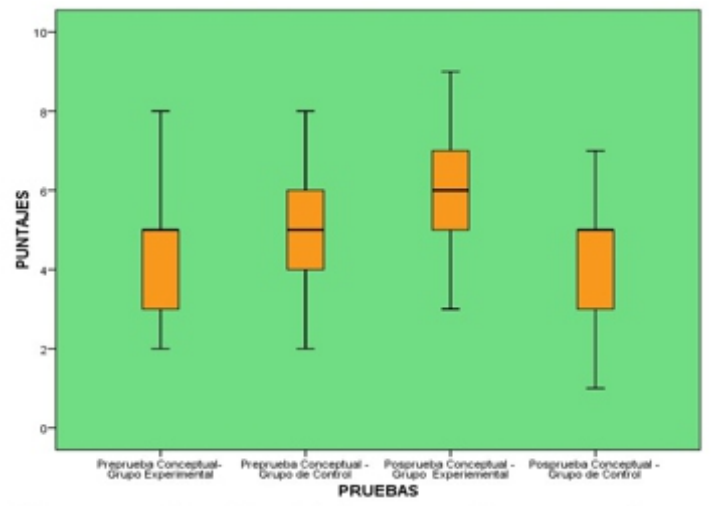

Figura 4. Rendimiento Conceptual en

\section{Cinemática.}

\section{Rendimiento Procedimental}

En los resultados estadísticos del Pre Prueba aplicada a los grupos de estudio, los conocimientos previos de los estudiantes antes de la aplicación del experimento, presentan resultados diferentes en cuanto al procedimiento en la solución de ejercicios de Cinemática, por lo que la significancia estadística $p=0,226>0,05=$, es distinto al $95 \%$ de confiabilidad de acuerdo a la prueba de Rangos de Wilcoxon. También se observa una diferencia en la media de 0,61 del Grupo Experimental respecto al Grupo de Control. Por lo tanto, no es significativa la diferencia entre ambos grupos.

Así mismo, observamos los resultados estadísticos de la Pos Prueba tomado a los grupos de estudio, los estudiantes a quienes se les aplicó el Modelo 4MAT obtuvieron notas superiores (Promedio $=4,56$ ) frente al Grupo de Control (Promedio= 2,51), de lo cual proviene el grado de significancia estadística $p=0$, $001<0,05=$, esto significa rechazar la hipótesis nula y aceptar la hipótesis alterna. También se observa una diferencia en la media de 2,05 del Grupo Experimental respecto al Grupo de Control. Por lo tanto, es significativa la diferencia entre ambos grupos. La figura 5 complementa estos resultados.

\section{Rendimiento Actitudinal}

Se observa en los resultados estadísticos de la Pre

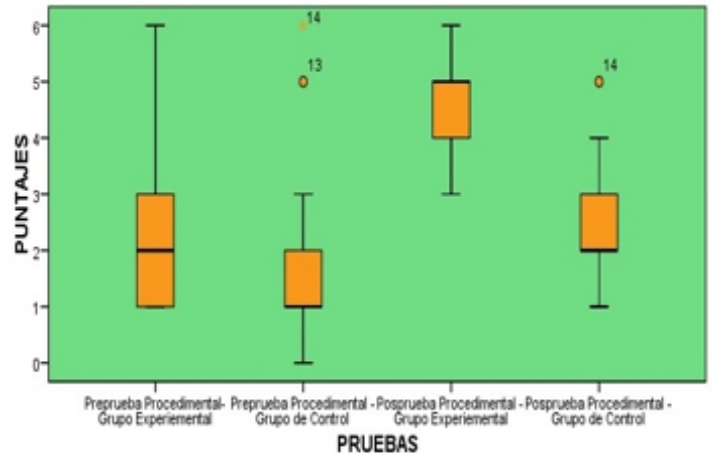

Figura 5. Rendimiento Procedimental en Cinemática.

Prueba evaluado a los grupos de estudio, Grupo Experimental y Grupo de Control, ligeramente diferentes respecto a su familiarización con el tema de Cinemática, por lo que el grado de significancia estadística $p=0,204>0,05=$, además $\left(t_{c}=1,322<\right.$ $\left.t_{t}=1,74\right)$, es distinto al $95 \%$ de confiabilidad de acuerdo a la prueba T de Student. También se observa una diferencia en la media de 1,7778 del Grupo Experimental respecto al Grupo de Control. Por lo tanto, no es significativa la diferencia entre ambos grupos.

Después de la aplicación del experimento el Grupo Experimental marco diferencia significativa frente al grupo de control con 14,00 puntos; lo que significa que los estudiantes del Grupo Experimental presentan puntuaciones por encima del Grupo Control en el cambio actitudinal. Del análisis de los resultados a varianzas iguales, frente a las comparaciones de los estadísticos $\left(t_{c}=20,698>t_{t}=1\right.$, 74), así mismo (T Student: $p=0,000<0,05=$ ) significa rechazar la hipótesis nula y aceptar la hipótesis alterna. Por lo tanto, es significativa la diferencia entre ambos grupos. De esta forma podemos ver que la aplicación del Modelo 4MAT influye en el cambio de Actitud en el aprendizaje de Cinemática en los estudiantes.

La Figura 6 complementa lo dicho anteriormente

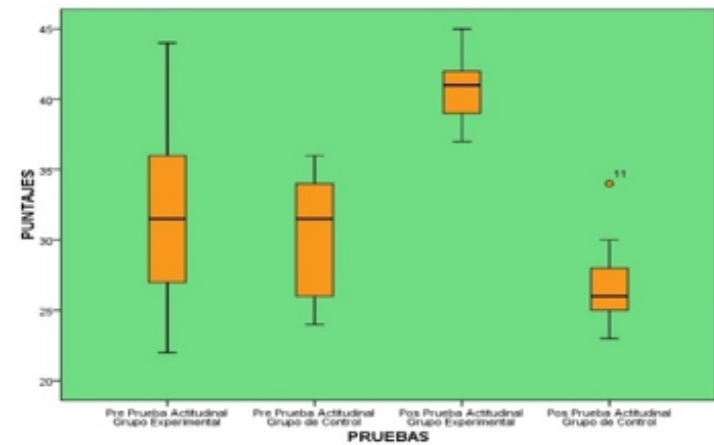

Figura 6. Rendimiento Actitudinal en Cinemática. 


\section{DISCUSIÓN}

Los resultados de la aplicación de pruebas (Pre Prueba y Pos Prueba), para el rendimiento académico, han sido analizado mediante la prueba estadística $\mathrm{T}$ de Student. Los estudiantes del grupo experimental obtuvieron mejores notas $($ Promedio $=$ 10,44) después de la aplicación del Modelo 4MAT respecto a los estudiantes del grupo de control (Promedio $=6,94)$; así mismo del grado de significancia estadística $\mathrm{p}=0,00<=0,05$, significa rechazar la hipótesis nula $\mathrm{H}_{0}$ y aceptar la hipótesis alterna $\mathrm{H}_{1}$. Caso contrario se hubiera aceptado la hipótesis nula $\mathrm{H}_{0}$.

De estos resultados estadísticos en la Pos Prueba en ambos grupos (Experimental y de Control) después del experimento, podemos decir, que la aplicación del Modelo 4MAT influye significativamente en el rendimiento académico en Cinemática de los estudiantes del Ciclo II de Ingeniería Agroindustrial. Así mismo, los resultados obtenidos indican que existen diferencias significativas entre los grupos de estudio en la el Pos Prueba, presentando el grupo experimental valores más altos que el grupo de control, por lo que podemos afirmar positivamente y aceptar lo propuesto en la hipótesis general de investigación: La aplicación del Modelo 4MAT influye en el rendimiento académico en Cinemática en los estudiantes.

De igual forma se demuestra que para las dimensiones: D1: Rendimiento Conceptual analizado mediante la prueba de $\mathrm{T}$ Student; Dimensión D2: Rendimiento Procedimental analizado mediante la prueba de Wilcoxon y la Dimensión D3: Rendimiento Actitudinal analizado mediante la prueba $\mathrm{T}$ de Student, que la aplicación del Modelo 4MAT influye significativamente en la mejora del rendimiento académico y el cambio actitudinal, respectivamente, de los estudiantes con estos resultados queda respaldada nuestra propuesta de aplicar el Modelo 4 MAT como estrategia de enseñanza en la mejora del rendimiento académico a nivel universitario.

Estos resultados se encuentra enmarcados en la línea de otros trabajos sustentados en (Artamónova, et al., 2014; Mohamed et al., 2016; Rosado, 2011; Silva, 2011; Ramírez, 2009;Pantoja, 2011; Marzano, 2014) en el sentido que utilizaron nuevas metodologías en la enseñanza aprendizaje con el objetivo de mejorar el nivel de rendimiento académico en los estudiantes o ver la correlación entre la aplicación de una nueva metodología y la forma como aprende el estudiante y mejora su rendimiento académico.

De los aspectos teóricos se resalta, que la gran preocupación de docentes de ciencia y especialmente de quienes enseñan Física, está en buscar nuevas alternativas, metodologías de enseñanza aprendizaje distintas de las tradicionales que enseñan en el marco de los modelos masivos. Se requiere de metodologías dinámicas en las cuales en el proceso de enseñanzaaprendizaje sea considerado el estilo, es decir, la forma como el individuo aprende y como procesa la información, de esa manera estaremos dándole un nuevo enfoque a la forma como se enseña, no solo decir que el aprendizaje está centrado en el estudiante y él hace solo su aprendizaje. Debe buscarse una metodología que considere a la enseñanza aprendizaje como un proceso, preocupándonos por su estilo particular de aprendizaje y haciendo que también domine otros estilos.

Por otro lado, debe contextualizarse esta problemática a quienes dirigen y establecen o definen las políticas y el modelo de enseñanza en las universidades, que deben cambiar su forma de brindar el servicio en el marco de la calidad y acreditación, deben preocuparse por mejorar, la infraestructura, laboratorios de docentes y de investigación, el uso de recursos TIC en las aulas y laboratorios, etc. También está la contextualización del trabajo de los docentes, se requiere que los docentes se actualicen en el uso de nuevas metodologías de enseñanza aprendizaje y en la actualización en el manejo de nuevas tecnologías como recurso de enseñanza, ya que la educación en su conjunto es un proceso dinámico.

Las limitaciones en la ejecución de esta investigación se encontrarían en que no se cuenta con adecuada infraestructura, laboratorios apropiados, bibliotecas no suficientemente implementadas, aulas carentes de los recursos TIC, etc., el tiempo para la ejecución de las actividades no es el óptimo, es necesario adecuar los planes curriculares. Por otro lado, la aplicación del Modelo 4MAT, aunque no como limitante en la realización de este trabajo si no como un aporte en futuros trabajos de investigación debe ser extendido a otras áreas (Matemática, Química, etc.) y con otros enfoques y diseños experimentales, el Modelo 4MAT debe ser actualizado de acuerdo con los avances de la Neurociencias en futuras investigaciones.

\section{CONCLUSIONES}

La aplicación del Modelo 4MAT influye significativamente en el rendimiento académico de estudiantes del curso de cinemática del ciclo II de Ingeniería Agroindustrial UNIA, esta aseveración se sustenta en el análisis estadístico de T de Student, pues los estudiantes del grupo experimental obtuvieron mejores notas (Promedio 10,44) después del experimento respecto a los estudiantes del grupo 
de control (Promedio $=5,89$ ); por valores de significancia estadística $\mathrm{p}=0,00<0,05=\alpha$ además, $(\mathrm{tc}=1,154>\mathrm{tt}=1,74)$, se aceptó la hipótesis de investigación.

Respecto al rendimiento conceptual en Cinemática, la aplicación del Modelo 4MAT influyó significativamente en el Rendimiento Académico de los estudiantes.

En consideración al rendimiento procedimental en Cinemática, se demuestra que la aplicación del Modelo 4MAT influye significativamente en el Rendimiento Académico de los estudiantes.

Se demuestra también, que la aplicación del Modelo 4MAT influye significativamente en cuanto al cambio actitudinal, en relación al aprendizaje de la Cinemática, en los estudiantes.

Finalmente, respecto a los conocimientos previos de los estudiantes del ciclo II de Ingeniería Agroindustrial UNIA 2017, antes de la aplicación del Modelo 4 MAT; se demuestra que no existen diferencias significativas según los resultados estadísticos de T de Student $p=0,929>0,05=$ además $\left(t_{c}=0,090<t_{t}=1,74\right)$, según los resultados de la Pre Prueba, lo que indica que ambos grupos propuestos (Experimental y de Control) de estudiantes del ciclo II de la Escuela de Ingeniería Agroindustrial son semejantes en sus conocimientos previos sobre Cinemática.

\section{REFERENCIAS BIBLIOGRÁFICAS}

Artomónova, I., Mosquera, J., Ramírez, M., y Mosquera, D. (2014). Resultados cuantitativos de la aplicación del Sistema 4MAT en Mecánica en la Universidad del Quindío. Latin American Journal of Physics Education, 8(4).

Dikkartin, F. (2012). The Effect of the 4MAT Model on Student's, Algebra Achievements and Level of Reaching Attainments. Int. J. Contemp. Math. Sciences, 7(45):2197-2205.

Hernández, R., Fernández, C., y Baptista, M. (2014). Metodología de la Investigacion. McGrawHill, 6ta edición.

Leonardo, E. y Tarazona, V. (2014). Relación de estilos de aprendizaje y el rendimiento académico de los alumnos de I ciclo de la Escuela de Mecánica de Fluidos de la Universidad Nacional Mayor de San Marcos en el curso de Cálculo Diferencial 2013-I. Tesis de Maestría, Universidad Tecnológica del Perú.
Marzano, R. (2014). Aplicación del Sistema multimedia interactivo (SAMI) en la enseñanza de Física para el logro de aprendizajes de los estudiantes de la Facultad de Ciencias, de la Universidad Nacional de Educación. "Enrique Guzmán y Valle". Tesis de Doctorado, Universidad San Martin de Porras.

McCarthy, B. y McCarthy, D. (2006). Teaching around the 4MAT (R) cycle: Designing Instruction for Diverse Learners with Diverse Learning styles. Thousand Oaks, United States.

Mendenhall, W., Beaver, R., y Beaver, B. (2010). Introducción a la Probabilidad y Estadística. Cengage, Learning. 13ava Edición.

Mohamed, O., Fahad, A., y Mohamed, A. (2016). Effect of using 4mat method on academic achievement and attitudes toward Engineering Economy for undergraduate students. International Journal of Vocational and Technical Education, 8(1), 1-11.

Mori, M. (2012). Deserción universitaria en estudiantes de una Universidad Privada de Iquitos. Revista Digital de Investigacion en Docencia Universitaria, Universidad Peruana de Ciencias Aplicadas, 1(1).

Muñoz, J., Gutiérrez, P., y Serrano, R. (2012). Los hemisferios cerebrales: dos estilos de pensar, dos modos de enseñar y aprender. Universidad de Córdoba.

Nicoll, J. y Seider, S. (2010). Assessing the Impact of the 4MAT Teaching Model Across Multiple Disciplines in Higher Education. Central Connecticut State University. College Teaching, 58:19-27.

Nowacki, A. (2011). Using the 4MAT Framework to Design Problem-Based Learning Biostatistics Course. Journal of Statistics Education, 19(3):4-6.

Pantoja, H. (2015). Aplicación del software libre SAGE y su influencia en el rendimiento académico en Cálculo Vectorial, en los estudiantes del IV ciclo de Ingeniería Mecánica de la Universidad Nacional de Ingeniería. Tesis de Doctorado, Universidad Nacional de Educación, Enrique Guzmán y Valle.

Ramírez, A., Rojas, L., Hidalgo, C., García, R., y Molinar, J. (2013). Relación entre estilos de aprendizaje, rendimiento académico y otras 
variables relevantes de estudiantes universitarios. Revista Estilos de Aprendizaje, 11(12):1-16.

Ramírez, M. (2009). Aplicación del Sistema 4MAT en la enseñanza de la Física a nivel universitario. Tesis de Doctorado, Instituto Politécnico Nacional-Colombia.

Ramírez, M. (2010). Aplicación del Sistema 4MAT en la enseñanza de la Física a nivel universitario. Revista Mexicana de Física, 56(1), 29-40.

Ramírez, M. y Chávez E. (2012). Similitudes del Sistema 4MAT de estilos de aprendizaje y la metodología de clases interactivas demostrativas en la enseñanza de la Física. Revista de Estilos de Aprendizaje. 9(9), 116.

Rosado, C. (2011). Aplicación de Sistema 4MAT en ambientes de aprendizaje mixto para la enseñanza de la Física a nivel Ingeniería. Tesis de Maestría, Instituto Politécnico Nacional, México.

Rosado, C. y Sánchez, D. (2012). The 4MAT system applied to a blended-learning scenario. Lat. Am. J. Phys. Educ., 6(1):275-279.

Segarra, M., Estrada, M., y Monferrer, D. (2015). Estilos de aprendizaje en estudiantes universitarios: lateralización vs interconexión de los hemisferios cerebrales. Revista española de pedagogía, 1(252):583-600.

Silva, R. (2011). La enseñanza de la Física mediante un aprendizaje significativo y cooperativo en Blended Learning. Tesis de doctorado, Universidad de Burgos.

Tatar, E. y Dikici, R. (2009). The effect of the 4MAT Method (learning styles and brain hemispheres) of instruction on achievement in Mathematics. International Journal of Mathematical, Education in Science and Technology, 40(8):1027-1036.

Vizconde, M. (2009). Programa de inteligencia emocional para incrementar el rendimiento académico en el área de comunicación integral de los alumnos del 5to. Grado de educación secundaria de la I.E.P. "Dante Alighieri”, Trujillo-2009. Tesis de Maestría, Universidad Nacional de Trujillo. 